\title{
Duality between disordered nodal semimetals and systems with power-law hopping
}

\author{
S. V. Syzranov ${ }^{1}$ and V. Gurarie ${ }^{2}$ \\ ${ }^{1}$ Physics Department, University of California, Santa Cruz, California 95064, USA \\ ${ }^{2}$ Department of Physics and Centre for Theory of Quantum Matter, University of Colorado, Boulder, Colorado 80309, USA
}

(Received 13 August 2019; published 11 December 2019)

\begin{abstract}
Nodal semimetals (e.g., Dirac, Weyl, and nodal-line semimetals, graphene, etc.) and systems of pinned particles with power-law interactions (trapped ultracold ions, nitrogen defects in diamonds, spins in solids, etc.) are presently at the center of attention of large communities of researchers working in condensed-matter and atomic, molecular, and optical physics. Although seemingly unrelated, both classes of systems are abundant with novel fundamental thermodynamic and transport phenomena. In this Rapid Communication, we demonstrate that low-energy field theories of quasiparticles in semimetals may be mapped exactly onto those of pinned particles with power-law hopping excitations. The duality between the two classes of systems, which we establish, trades strong disorder in one class for weak disorder in the other, and allows one to describe the transport and thermodynamics of each class of systems using the results established for the other class. In particular, using the duality mapping, we establish the existence of another class of disorder-driven transition in systems with the powerlaw hopping $\propto 1 / r^{\gamma}$ of excitations with $d / 2<\gamma<d$, different from the conventional Anderson-localization transition. Non-Anderson disorder-driven transitions have been studied broadly for nodal semimetals. Our work extends the class of systems exhibiting such transitions to systems with long-range hopping (interactions) with $\gamma<d$.
\end{abstract}

DOI: 10.1103/PhysRevResearch.1.032035

The last few years have seen an explosion of interest in nodal semimetals, such as Weyl, parabolic, and nodal-line semimetals [1], owing to their potential applications in future electronic and spintronic devices, in addition to the abundance of novel fundamental phenomena observed in these materials: chiral anomaly [2-6], magnetohydrodynamic effects [7-10], topologically protected surface states [11-13], etc. These systems have also changed the current perspective on phase transitions in disordered systems; Weyl and Dirac semimetals have been demonstrated to display disorder-driven phase transitions (or possibly very sharp crossovers [14]) in universality classes different from those of the Anderson metal-insulator transitions [15-17]. These transitions have been demonstrated [18] to occur also in a broader class of systems in sufficiently high dimensions.

Another, seemingly unrelated, class of systems, which has recently attracted attention, are systems of pinned particles with power-law interactions. The amplitude of hopping of excitations in these systems displays a power-lay decay with distance, $\propto 1 / r^{\gamma}$. Such systems include, but are not limited to, polar molecules $[19,20](\gamma=3)$, impurity spins in solids, Rydberg atoms [21] $(\gamma=3$ or $\gamma=6)$, nitrogen vacancies in diamonds $[22,23](\gamma=3)$, and neutral excitations in strongly disordered electronic systems $(\gamma=3$ in 3D systems and $\gamma=$ 2 in thin dielectric films [24-26]). Furthermore, power-law

Published by the American Physical Society under the terms of the Creative Commons Attribution 4.0 International license. Further distribution of this work must maintain attribution to the author(s) and the published article's title, journal citation, and DOI. hopping with a tunable parameter $0<\gamma<3$ has been realized [27-30] recently in one- and two-dimensional (1D and 2D) systems of trapped ultracold ions. Similarly to the case of nodal semimetals, in the presence of quenched disorder, power-law hopping is expected to lead to unconventional localization phenomena or disorder-driven criticality (see, e.g., Refs. [31-40]) and is also studied often in the context of many-body localization [41-46].

Nodal semimetals and systems with power-law hopping may seem completely unrelated and are usually studied independently by two communities of researchers working, respectively, on condensed-matter systems and in atomic, molecular, and optical physics. As it has already been noted $[17,38]$, however, $d$-dimensional systems with power-law hopping, whose amplitude decays with distance as $\propto 1 / r^{\gamma}$ with $d<\gamma<3 d / 2$, display the same type of non-Anderson disorder-driven transitions which take place in nodal semimetals, and which are absent for faster decay, corresponding to $\gamma>3 d / 2$. There is thus a natural connection between the two classes of systems. The phenomenology of systems with slower decay of the hopping, corresponding to $0<\gamma<d$, is understood, in our opinion, much more poorly; the possibility of the unconventional disorder-driven transitions in them needs to be investigated.

In this Rapid Communication, we derive a duality transformation between the field theories of disordered systems with the power-law dispersion $\alpha k^{\alpha}$, with $0<\alpha<d$, typical for nodal semimetals, and the field theories of hopping in arrays of random-energy sites with the amplitude $\propto 1 / r^{\gamma}$, with $0<\gamma<d$, hereinafter referred to as long-range hopping (as opposed to short-range hopping, decaying with distance faster than $\left.1 / r^{d}\right)$. Since particles displaying the long-range hopping 


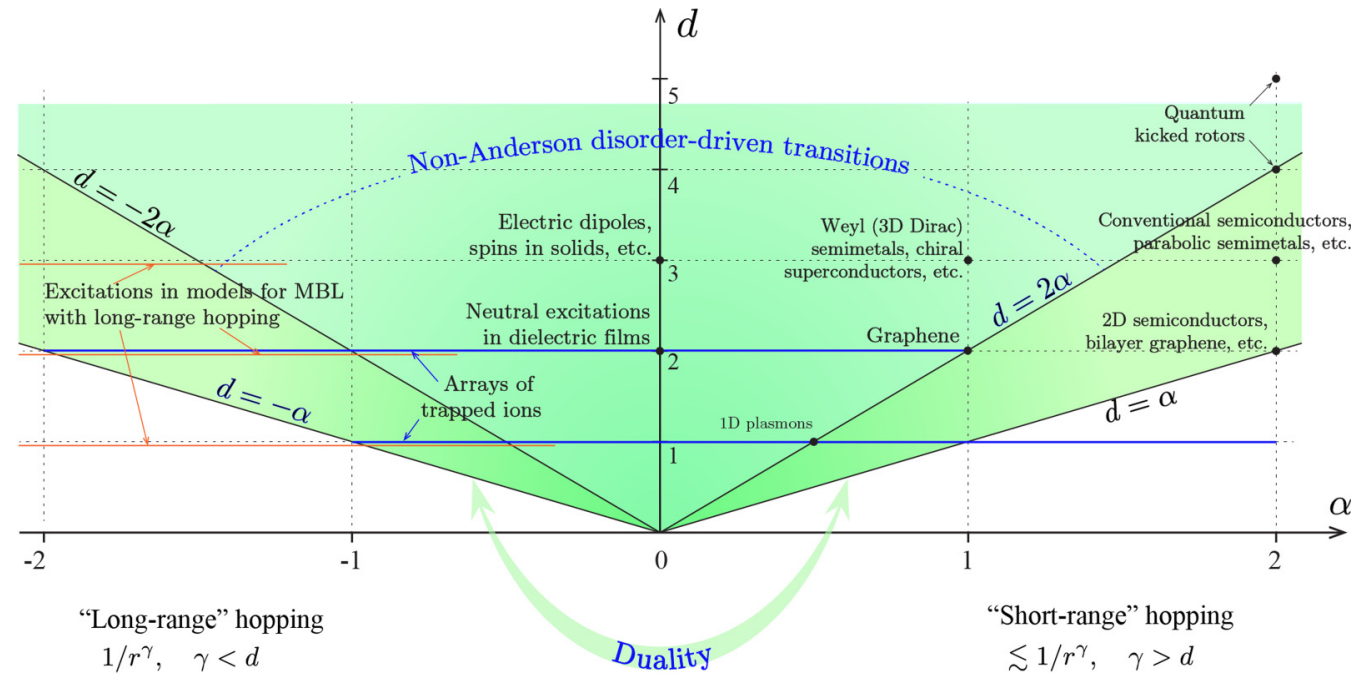

FIG. 1. Examples of systems with the power-law dispersion $\xi_{\mathbf{k}} \propto k^{\alpha}$ in the "dimension $d$ vs $\alpha$ diagram." $\alpha>0$ corresponds to nodal semimetals and systems where hopping decays with distance faster than $1 / r^{d}$. Systems with $\alpha<0$ are represented by trapped ultracold particles with long-range interactions and models of many-body localization (MBL) transitions with power-law hopping. The duality transformation, described in this Rapid Communication, maps the region $d>\alpha>0$ of the diagram onto the region $d>-\alpha>0$. This duality allows us to predict the disorder-driven phase transition in systems with long-range hopping, which correspond to $d>-2 \alpha>0$.

between sites may be considered as having a singular kinetic energy $\propto k^{\gamma-d}$, the duality transformation may be said to establish a mapping between disordered systems with kinetic energies $k^{\alpha}$ and $k^{-\alpha}$, as shown in Fig. 1, where $0<\alpha=$ $\gamma-d<d$. It is of note that this duality exchanges strong and weak disorder in the two phases it connects. This duality mapping may be used to describe phenomena in each of these two classes of systems, by using theoretical descriptions and experimental results available for the other class, or to develop new models and descriptions.

Using the established duality, we predict another class of disorder-driven quantum phase transitions in systems with long-range hopping $\propto 1 / r^{\gamma}$ with $d / 2<\gamma<d$. These transitions are dual to the non-Anderson disorder-driven transitions [17] in nodal semimetals and systems with short-range hopping (see Fig. 1). While unconventional disorder-driven transitions have been a target of vigorous studies in the context of Weyl semimetals (see Ref. [17] for a review) and of shortrange hopping [35-38,47] (i.e., decaying faster than $1 / r^{d}$ ), disorder-driven transitions for long-range hopping $\propto 1 / r^{\gamma}$, with $\gamma<d$, are established here by means of the duality arguments.

These transitions manifest themselves in the singular behavior of the density of states (DOS) and other observables. We emphasize that these transitions are not accompanied by localization or delocalization of wave functions, as all states are expected to be delocalized for long-range hopping [31,33], and belong to a universality class different from that $[48,49]$ of Anderson localization. These transitions may be realized by means of ultracold ions in optical or magnetic traps [27-30] and also occur for excitations in certain models which are used usually for studying many-body localization-delocalization transitions in the presence of power-law hopping [45,46,50].

Duality mapping. To establish the duality, we consider a semimetal with the power-law quasiparticle dispersion $\xi=a k^{\alpha}$ near the node, which has a trivial spin and sublattice structure, in the presence of randomly located short-range impurities, whose potentials may be approximated by delta functions with amplitudes $\lambda_{n}, u(\mathbf{r})=\sum_{n} \lambda_{n} \delta\left(\mathbf{r}-\mathbf{r}_{n}\right)$. Our arguments may easily be generalized to the cases of more complicated dispersions, including spin and valley structures, and impurity potentials [51]. In this Rapid Communication, we focus on single-particle disorder-driven phenomena and use a supersymmetric [49] field theory to describe quasiparticle states whose action is given by

$$
\begin{aligned}
\mathcal{L}_{\mathrm{SM}}= & -i \int \bar{\psi}\left[E-a|\hat{\mathbf{k}}|^{\alpha}+i 0 \Lambda\right] \psi d \mathbf{r} \\
& +i \sum_{n} \lambda_{n} \bar{\psi}\left(\mathbf{r}_{n}\right) \psi\left(\mathbf{r}_{n}\right),
\end{aligned}
$$

where $\hat{\mathbf{k}}=-i \partial_{\mathbf{r}}$ is the momentum operator; $\psi$ is a fourcomponent supervector with components in the $\mathrm{BF} \otimes \mathrm{RA}$ (boson-fermion $\otimes$ retarded-advanced) space [49], $\Lambda=\mathbb{1}_{\mathrm{BF}} \otimes$ $\left(\hat{\sigma}_{z}\right)_{\mathrm{RA}}$, and $\bar{\psi}=\psi^{\dagger} \Lambda$.

Values of observables in this semimetal may be represented in the form $\langle\mathcal{O}\rangle=\int D \psi D \psi^{\dagger} \cdots e^{-\mathcal{L}_{\mathrm{SM}}}$, where "..." are supersymmetry-breaking terms. Introducing supervectors $\phi\left(\mathbf{r}_{n}\right)$ at the locations $\mathbf{r}_{n}$ of the impurities by means of the Habbard-Stratonovich transformation $e^{-i \lambda \bar{\psi} \psi}=$ $\int \mathcal{D} \phi^{\dagger} \mathcal{D} \phi e^{i \frac{\phi \phi-\lambda \psi \phi-\lambda \phi \psi}{\lambda-i 0 \Lambda}}$ and integrating out the fields $\bar{\psi}$ and $\psi$ leads to a field theory with the action

$$
\mathcal{L}=i \sum_{m, n} \bar{\phi}\left(\mathbf{r}_{n}\right) G_{E}\left(\mathbf{r}_{n}, \mathbf{r}_{m}\right) \phi\left(\mathbf{r}_{m}\right)-i \sum_{n} \frac{\bar{\phi}\left(\mathbf{r}_{n}\right) \phi\left(\mathbf{r}_{n}\right)}{\lambda_{n}-i 0 \Lambda},
$$

in terms of the fields $\phi$, where $G_{E}=\int \frac{d \mathbf{k}}{(2 \pi)^{d}} \frac{e^{-i \mathbf{k r}}}{E-a|\mathbf{k}|^{\alpha}+i 0 \Lambda}$ is a matrix of the Green's functions in a disorder-free semimetal.

In the vast majority of nodal semimetals (such as 3D Dirac, Weyl, and parabolic semimetals, graphene) the exponent $\alpha$ 
of the dispersion $\xi_{\mathbf{k}} \propto|\mathbf{k}|^{\alpha}$ is exceeded by the dimensions $d$. Power-law dispersion with $\alpha<d$ may also be realized in $1 \mathrm{D}$ and $2 \mathrm{D}$ systems of trapped ultracold ions [27-30] and certain other systems, such as 1D plasmons (corresponding to $\alpha=1 / 2)$. In all these systems, the Green's functions $G_{E}(\mathbf{r})$ at low energies $E$ display power-law decay with distance $r$, which gives the action

$$
\mathcal{L}_{\text {Hop }}=-i \sum_{m, n} \bar{\phi}\left(\mathbf{r}_{n}\right)\left[\frac{j}{\left|\mathbf{r}_{n}-\mathbf{r}_{m}\right|^{d-\alpha}}+\delta_{n m} \lambda_{n}^{-1}+i 0 \Lambda\right] \phi\left(\mathbf{r}_{m}\right),
$$

where $j=\frac{\Gamma\left(\frac{d-\alpha}{2}\right)}{2^{\alpha} a \pi^{\frac{d}{2}} \Gamma\left(\frac{\alpha}{2}\right)}$ for the case of the dispersion $\xi_{\mathbf{k}}=$ $a|\mathbf{k}|^{\alpha}$ with a trivial spin and valley structure considered here. For more complicated structures of the dispersion of the semimetal, the first term in the action (3) will still display the power-law decay $\propto 1 /\left|\mathbf{r}_{n}-\mathbf{r}_{m}\right|^{d-\alpha}$, but will involve the respective valley or spin degrees of freedom. For example, in the case of a Weyl semimetal, with the quasiparticle dispersion given by $\xi_{\mathbf{k}}=v \hat{\boldsymbol{\sigma}} \cdot \mathbf{k}$, where $\hat{\boldsymbol{\sigma}}$ is the pseudospin operator, the system may be described by action (3) with the replacement $j \rightarrow-\frac{1}{8 \pi} \frac{\hat{\sigma} \cdot\left(\mathbf{r}_{n}-\mathbf{r}_{m}\right)}{v\left|\mathbf{r}_{n}-\mathbf{r}_{m}\right|^{3}}$.

Action (3) describes particle hopping between randomly located sites, with the energies $-\lambda_{n}^{-1}$, where the hopping amplitude decays with distance as the power law $\propto 1 / \mid \mathbf{r}_{n}-$ $\left.\mathbf{r}_{m}\right|^{d-\alpha}$. The transformation from action (1) to action (3) is, therefore, a duality transformation between the field theories of a disordered nodal semimetal and a systems with power-law hopping in an array of random-energy sites.

This duality may be used to explore novel phenomena in each of these two classes of systems using the theories and experimental results available for the other class. In particular, we predict in what follows a disorder-driven quantum phase transition for systems with power-law hopping, which is dual to the disorder-driven transition in semimetals in high dimensions [17].

Non-Anderson disorder-driven transitions in systems with long-range hopping. In order to elucidate these transitions, we consider a spin-1/2XY model on randomly located sites, where the exchange interaction between the spins depends on the distance between them as the power law. The Hamiltonian of this model, which may be realized by means of trapped ultracold particles, is given by

$$
\hat{\mathcal{H}}=\sum_{n} E_{n} \hat{s}_{n}^{z}-\sum_{n, m} \frac{j}{\left|\mathbf{r}_{n}-\mathbf{r}_{m}\right|^{\nu}}\left(\hat{s}_{n}^{+} \hat{s}_{m}^{-}+\hat{s}_{m}^{+} \hat{s}_{n}^{-}\right),
$$

where the on-site magnetic fields $-E_{n}$ are random and uncorrelated on different sites and we consider $\gamma<d$.

In general, in the limit of small $j$, spin-flip-type excitations in this model propagate similarly to single particles with the amplitudes which decay with distance as the power law $[25,26,40,50,52]$. For simplicity, we assume that the on-site field $E_{n}$ fluctuates weakly on top of a large average value $\left\langle E_{n}\right\rangle_{\text {dis }}$ and, as a result, all the spins are almost entirely polarized in the same direction in the ground state. The propagation of spin-flip excitations may then be mapped exactly onto a single-particle model with the action (3).
In what follows, we demonstrate that the system exhibits a non-Anderson phase transition when changing the coupling amplitude $j$ or the amplitude of the fluctuations of the on-site energies $E_{n}$. This transition may manifest itself in the form of singularities in various physical observables, such as the diffusion coefficient or magnetic susceptibility. In what follows we focus on the behavior of the DOS of low-energy excitations.

Below, we consider the states of the Hamiltonian (4) with energies $E=\left\langle E_{n}\right\rangle_{\text {dis }}+\omega$ weakly deviating from the large average on-site field $\left\langle E_{n}\right\rangle_{\text {dis }}$. The DOS of single spin-flip excitations at energy $\omega$ is given by

$$
\begin{aligned}
\rho_{\mathrm{Hop}}(\omega) & =-\frac{1}{\pi V} \operatorname{Im} \partial_{\eta}\left\langle\int \mathcal{D} \phi \mathcal{D} \phi^{\dagger} e^{-\mathcal{L}_{\mathrm{Hop}}-\mathcal{L}_{\omega}-\mathcal{L}_{s}(\eta)}\right\rangle_{\mathrm{dis}}, \\
\mathcal{L}_{\omega} & =-i \omega \sum_{n} \bar{\phi}\left(\mathbf{r}_{n}\right) \phi\left(\mathbf{r}_{n}\right), \\
\mathcal{L}_{s}(\eta) & =i \eta \sum_{n} s_{R}^{*}\left(\mathbf{r}_{n}\right) s_{R}\left(\mathbf{r}_{n}\right),
\end{aligned}
$$

where $s_{R}\left(\mathbf{r}_{n}\right)$ is the retarded bosonic component of the supervector $\phi\left(\mathbf{r}_{n}\right) ; V$ is the volume of the system; $\mathcal{L}_{\text {Hop }}$ is given by Eq. (3) with $\lambda_{n}^{-1}=-E_{n}+\left\langle E_{n}\right\rangle_{\mathrm{dis}}$; the term $\mathcal{L}_{\omega}$ accounts for the effect of the energy $\omega$ on the action; $\mathcal{L}_{s}(\eta)$ is the supersymmetry-breaking source term; and $\eta$ is an infinitesimal parameter.

The full action $\mathcal{L}=\mathcal{L}_{\text {Hop }}+\mathcal{L}_{\omega}+\mathcal{L}_{s}(\eta)$ used to obtain the DOS (5a) thus matches action (3) with the replacement $\lambda_{n}^{-1} \rightarrow-E_{n}+\left\langle E_{n}\right\rangle_{\mathrm{dis}}+\omega-\eta \cdot \mathbb{1}_{B B R R}$, where $\mathbb{1}_{B B R R}$ is the projector to the bosonic retarded parts of supervectors. The corresponding dual action of a semimetal is given by Eq. (1) with the same replacement and with $\alpha=d-\gamma$ and $a=\frac{\Gamma\left(\frac{\gamma}{2}\right)}{2^{d-\gamma} \pi^{\frac{d}{2}} \Gamma\left(\frac{d-\gamma}{2}\right) j}$.

Expanding that action to the first order in small parameters $\omega$ and $\eta$ and performing disorder averaging of the DOS, while keeping only the first cumulants in the disorder strength

$$
\varkappa=N\left\langle\left(E_{n}-\left\langle E_{n}\right\rangle\right)^{-2}\right\rangle_{\mathrm{dis}},
$$

gives the effective action of a semimetal

$$
\begin{aligned}
\mathcal{L}_{\mathrm{SM}}= & -i \int \bar{\psi}\left[\omega \varkappa-a|\hat{\mathbf{k}}|^{d-\gamma}+i 0 \Lambda\right] \psi d \mathbf{r} \\
& +\frac{1}{2} \varkappa \int[\bar{\psi} \psi]^{2} d \mathbf{r}+i \eta \varkappa \int \tilde{s}_{R}^{*} \tilde{s}_{R} d \mathbf{r},
\end{aligned}
$$

where $\tilde{s}_{R}^{*}$ and $\tilde{s}_{R}$ are the retarded bosonic components of the supervectors $\bar{\psi}$ and $\psi$. We emphasize that we assume, when deriving Eq. (7), that the variance $\varkappa^{2} / N$ of the quantity $\left(E_{n}-\left\langle E_{n}\right\rangle\right)^{-1}$ is finite. For example, this quantity may be a random Gaussian variable, in which case the fluctuations of the energies $E_{n}$ are non-Gaussian; the statistics or even the existence of the variance of the energies $E_{n}$ have no bearing on our arguments.

Equation (7) is the action of a disordered semimetal with the quasiparticle dispersion $\xi_{\mathbf{k}}=a k^{d-\gamma}$ and with a source 
term which generates the DOS. According to Eq. (5a) and the action (7), the DOS of low-energy excitations in the model described by the Hamiltonian (4) is given by

$$
\rho_{\mathrm{Hop}}(\omega)=\varkappa \rho_{\mathrm{SM}}(\omega \varkappa)
$$

where $\rho_{\mathrm{SM}}(\omega \varkappa)$ is the DOS in the dual semimetal described by the action (7) at energy $\omega \varkappa$ and the disorder strength $\varkappa$ given by Eq. (6).

Semimetals described by the action of the form (7) (Dirac semimetals, high-dimensional semiconductors, quantum kicked rotors, etc.) are known to display phase transitions (or sharp crossovers [14]) between strong-disorder and weakdisorder phases at a certain value $\omega_{c}$ of the energy, hereinafter set to zero, and disorder strength. The relation (8) between the densities of states of a semimetal and a model with power-law hopping indicates that a disordered system with long-range hopping exhibits a critical scaling in the same universality class. However, because the on-site energies $E_{n}$ in the hopping model match the inverse amplitudes $\lambda_{n}^{-1}$ of the impurity potentials in the dual semimetal, the strong- and weak-disorder phases of the semimetal correspond, respectively, to the weak- and strong-disorder phases of a system with long-range hopping. If the disorder strength $\varkappa$ exceeds a critical value $\varkappa_{c}$ in a system with long-range hopping, or the coupling $j$ is smaller than a critical coupling $j_{c}$, the system is in a phase with a suppressed DOS of low-energy excitations. At weaker disorder or larger intersite couplings, the systems exhibit a continuous transition [14] to a phase with a larger DOS and better transport of the low-energy excitations.

Using Eq. (8) and the critical scaling proposed in Ref. [53] for a Dirac semimetal and derived microscopically in Ref. [54] for a generic semimetal with the power-law quasiparticle dispersion $\xi_{\mathbf{k}} \propto k^{\alpha}$, we find that the DOS near the transition in a system with long-range hopping has the form

$$
\rho_{\text {Hop }}(\omega, j)=\omega^{\frac{d}{z}-1} \Phi\left(\omega /\left|j-j_{c}\right|^{z v}\right)+\rho_{\text {smooth }},
$$

where $\Phi(x)$ is a universal scaling function which may be different for different signs of the argument, and $v$ and $z$ are the correlation length and the dynamical critical exponents (matching those of the dual transition in a semimetal $[17,36,55-59]) ; \rho_{\text {smooth }}$ is an analytic contribution which comes from the instantons in actions (1) and (3) ("rare-region effects" [54,60-66]).

In semimetals, the instantonic contribution $\rho_{\text {smooth }}$ to the DOS may be exponentially suppressed by the small deviation $\left|d-d_{c}\right|$ of the dimension $d$ from the critical dimension $d_{c}=$ $2 \gamma$ of the transition or the number of the particle flavours [17]. Even in the absence of small parameters, various numerical studies of 3D Weyl and Dirac semimetals have found this contribution to be rather small or unobservable $[17,53,56-$ 58,67-70], which allows one to use the DOS, to a good approximation, as an order parameter for the transition. It has also been suggested [14] that the instantonic contribution may broaden criticality, thus converting the transition to a sharp crossover. In this Rapid Communication, however, we do not distinguish between such sharp crossovers and phase transitions. The behaviors of the DOS for various values of

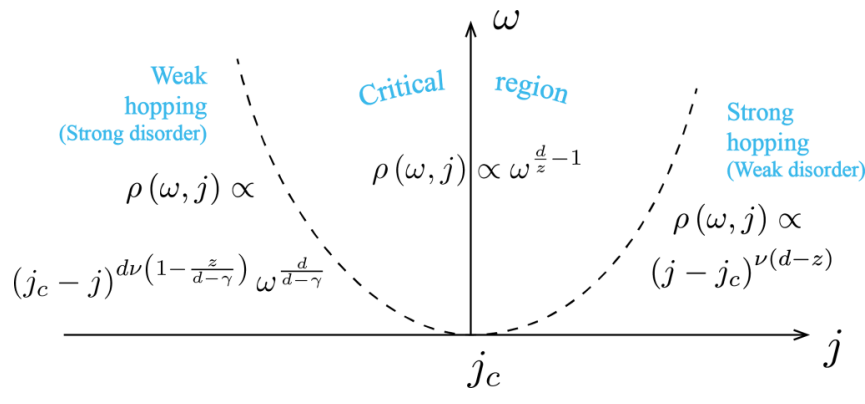

FIG. 2. Critical behavior of the density of states of low-energy excitations in a system with the power-law hopping $\propto j / r^{\gamma}$ in the diagram "excitation energy $\omega$ vs coupling $j$," where the energy $\omega$ is measured from the average potential. The same diagram with the replacement $j \rightarrow 1 / \varkappa$ describes the dependence of the DOS on energy $\omega$ and the disorder strength $\varkappa$.

the coupling $j$ and the excitation energies $\omega$ near the critical point are summarized in Fig. 2 and follow directly from the scaling form (9) and may also be inferred from the respective results for semimetals [17,53].

Experimental observation. The DOS of excitations in a spin model with the Hamiltonian (4) may be observed explicitly in experiments on trapped ultracold particles by measuring the spin susceptibility $\chi_{x x}(\omega)=$ $i \sum_{j} \int_{0}^{\infty}\left\langle\left[\hat{s}_{i}^{x}(t), \hat{s}_{j}^{x}(0)\right]\right\rangle e^{i t(\omega+i 0)} d t$. The imaginary part of the susceptibility determines the dissipation in the system due to creating spin-flip excitations and is related to the density of states of the excitations as $\operatorname{Im} \chi_{x x}(\omega)=N \rho(\omega)$.

Describing spin-flip excitations in the spin model with the Hamiltonian (4) by a single-particle model with action (3), considered here, is justified if all the spins are polarized in the ground state by the average value of the on-site magnetic magnetic field. It is also expected often that singleparticle descriptions for excitations in the considered spin model are appropriate even if the sign of the magnetic field fluctuates in space, so long as the parameter $j$ is sufficiently small $[25,26,40,50,52]$. In the latter case, we still expect the existence of disorder-driven phase transitions between the "weak-hopping" (irrelevant ratio of the hopping to disorder amplitudes) phase at small $j$ and a "strong-hopping" phase at larger $j$. We leave, however, such models for future studies.

Outlook. Natural further research directions include extending the duality between nodal semimetals and systems with long-range hopping, which we have established here, to interacting systems, more generic and anisotropic dispersions (e.g., in nodal-line and nodal-surface semimetals), and other models of disorder. The approach of describing semimetals in the dual representation, established here, may also be used to investigate, for example, rare-region effects and the possibility of other unconventional phase transitions.

Acknowledgments. We are grateful to L. Radzihovsky and B. Sbeirski for useful discussions and feedback on the manuscript. Also, we thank L. Radzihovsky for prior collaboration on related topics. Our work has also been supported by the Hellman Foundation (S.V.S.) and the Faculty Research Grant awarded by the Committee on Research from the University of California, Santa Cruz (S.V.S.). 
[1] N. P. Armitage, E. J. Mele, and A. Vishwanath, Weyl and Dirac semimetals in three-dimensional solids, Rev. Mod. Phys. 90, 015001 (2018).

[2] A. A. Burkov, Weyl metals, Annu. Rev. Condens. Matter Phys. 9, 359 (2018).

[3] S. A. Parameswaran, T. Grover, D. A. Abanin, D. A. Pesin, and A. Vishwanath, Probing the Chiral Anomaly with Nonlocal Transport in Three-Dimensional Topological Semimetals, Phys. Rev. X 4, 031035 (2014).

[4] X. Huang, L. Zhao, Y. Long, P. Wang, D. Chen, Z. Yang, H. Liang, M. Xue, H. Weng, Z. Fang, X. Dai, and G. Chen, Observation of the Chiral-Anomaly-Induced Negative Magnetoresistance in 3D Weyl Semimetal TaAs, Phys. Rev. X 5, 031023 (2015).

[5] S. Liang, J. Lin, S. Kushwaha, J. Xing, Ni Ni, R. J. Cava, and N. P. Ong, Experimental Tests of the Chiral Anomaly Magnetoresistance in the Dirac-Weyl Semimetals $\mathrm{Na}_{3} \mathrm{Bi}$ and GdPtBi, Phys. Rev. X 8, 031002 (2018).

[6] A. A. Burkov, Chiral Anomaly and Diffusive Magnetotransport in Weyl Metals, Phys. Rev. Lett. 113, 247203 (2014).

[7] E. V. Gorbar, V. A. Miransky, I. A. Shovkovy, and P. O. Sukhachov, Consistent hydrodynamic theory of chiral electrons in Weyl semimetals, Phys. Rev. B 97, 121105(R) (2018).

[8] A. Lucas, R. A. Davison, and S. Sachdev, Hydrodynamic theory of thermoelectric transport and negative magnetoresistance in Weyl semimetals, Proc. Natl. Acad. Sci. USA 113, 9463 (2016).

[9] N. Yamamoto, Chiral transport of neutrinos in supernovae: Neutrino-induced fluid helicity and helical plasma instability, Phys. Rev. D 93, 065017 (2016).

[10] V. Galitski, M. Kargarian, and S. Syzranov, Dynamo Effect and Turbulence in Hydrodynamic Weyl Metals, Phys. Rev. Lett. 121, 176603 (2018).

[11] X. Wan, A. M. Turner, A. Vishwanath, and S. Y. Savrasov, Topological semimetal and Fermi-arc surface states in the electronic structure of pyrochlore iridates, Phys. Rev. B 83, 205101 (2011).

[12] H. Weng, Y. Liang, Q. Xu, R. Yu, Z. Fang, Xi Dai, and Y. Kawazoe, Topological node-line semimetal in threedimensional graphene networks, Phys. Rev. B 92, 045108 (2015).

[13] S. Jia, S.-Y. Xu, and M. Z. Hasan, Weyl semimetals, Fermi arcs and chiral anomalies, Nat. Mater. 15, 1140 (2016).

[14] It is still being debated in the literature (see, e.g., Refs. [57,6266]) whether the respective transition in a Weyl semimetal is a genuine phase transition or a sharp crossover in physical observables. In this Rapid Communication we do not distinguish between transitions and sharp crossovers, so long as there exists a parametrically large interval of observables where the critical scaling is observed.

[15] E. Fradkin, Critical behavior of disordered degenerate semiconductors. I. Models, symmetries, and formalism, Phys. Rev. B 33, 3257 (1986).

[16] E. Fradkin, Critical behavior of disordered degenerate semiconductors. II. Spectrum and transport properties in mean-field theory, Phys. Rev. B 33, 3263 (1986).

[17] S. V. Syzranov and L. Radzihovsky, High-dimensional disorder-driven phenomena in Weyl semimetals, semiconductors, and related systems, Annu. Rev. Condens. Matter Phys. 9, 33 (2018).
[18] S. V. Syzranov, L. Radzihovsky, and V. Gurarie, Critical Transport in Weakly Disordered Semiconductors and Semimetals, Phys. Rev. Lett. 114, 166601 (2015).

[19] B. Yan, S. A. Moses, B. Gadway, J. P. Covey, K. R. A. Hazzard, A. M. Rey, D. S. Jin, and J. Ye, Observation of dipolar spinexchange interactions with lattice-confined polar molecules, Nature (London) 501, 521 (2013).

[20] T. Lahaye, C. Menotti, L. Santos, M. Lewenstein, and T. Pfau, The physics of dipolar bosonic quantum gases, Rep. Prog. Phys. 72, 126401 (2009).

[21] M. Saffman, T. G. Walker, and K. Mølmer, Quantum information with Rydberg atoms, Rev. Mod. Phys. 82, 2313 (2010).

[22] M. V. G. Dutt, L. Childress, L. Jiang, E. Togan, J. Maze, F. Jelezko, A. S. Zibrov, P. R. Hemmer, and M. D. Lukin, Quantum register based on individual electronic and nuclear spin qubits in diamond, Science 316, 1312 (2007).

[23] G. Waldherr, Y. Wang, S. Zaiser, M. Jamali, T. SchulteHerbrüggen, H. Abe, T. Ohshima, J. Isoya, J. F. Du, P. Neumann, and J. Wrachtrup, Quantum error correction in a solid-state hybrid spin register, Nature (London) 506, 204 (2014).

[24] J. E. Mooij and G. Schön, Single charges in 2-dimensional junction arrays, in Single Charge Tunneling, edited by H. Grabert and M. H. Devoret (Plenum, New York, 1992).

[25] I. L. Aleiner, B. L. Altshuler, and K. B. Efetov, Localization and Critical Diffusion of Quantum Dipoles in Two Dimensions, Phys. Rev. Lett. 107, 076401 (2011).

[26] P. Titum, V. L. Quito, and S. V. Syzranov, Energy-level statistics in strongly disordered systems with power-law hopping, Phys. Rev. B 98, 014201 (2018).

[27] P. Richerme, Z.-X. Gong, A. Lee, C. Senko, J. Smith, M. FossFeig, S. Michalakis, A. V. Gorshkov, and C. Monroe, Non-local propagation of correlations in quantum systems with long-range interactions, Nat. Lett. 511, 198 (2014).

[28] R. Islam, C. Senko, W. C. Campbell, S. Korenblit, J. Smith, A. Lee, E. E. Edwards, C.-C. J. Wang, J. K. Freericks, and C. Monroe, Emergence and frustration of magnetism with variable-range interactions in a quantum simulator, Science 340, 583 (2013).

[29] P. Jurcevic, B. P. Lanyon, P. Hauke, C. Hempel, P. Zoller, R. Blatt, and C. F. Roos, Quasiparticle engineering and entanglement propagation in a quantum many-body system, Nature (London) 511, 202 (2014).

[30] P. Jurcevic, P. Hauke, C. Maier, C. Hempel, B. P. Lanyon, R. Blatt, and C. F. Roos, Spectroscopy of Interacting Quasiparticles in Trapped Ions, Phys. Rev. Lett. 115, 100501 (2015).

[31] L. S. Levitov, Absence of localization of vibrational modes due to dipole-dipole interaction, Europhys. Lett. 9, 83 (1989).

[32] L. S. Levitov, Critical Hamiltonians with long range hopping, Ann. Phys. 511, 697 (1999).

[33] L. S. Levitov, Delocalization of Vibrational Modes Caused by Electric Dipole Interaction, Phys. Rev. Lett. 64, 547 (1990).

[34] X. Deng, V. E. Kravtsov, G. V. Shlyapnikov, and L. Santos, Duality in Power-Law Localization in Disordered One-Dimensional Systems, Phys. Rev. Lett. 120, 110602 (2018).

[35] A. Rodríguez, V. A. Malyshev, G. Sierra, M. A. MartínDelgado, J. Rodríguez-Laguna, and F. Domínguez-Adame, Anderson Transition in Low-Dimensional Disordered Systems 
Driven by Long-Range Nonrandom Hopping, Phys. Rev. Lett. 90, 027404 (2003).

[36] A. V. Malyshev, V. A. Malyshev, and F. Domínguez-Adame, Monitoring the localization-delocalization transition within a one-dimensional model with nonrandom long-range interaction, Phys. Rev. B 70, 172202 (2004).

[37] F. A. B. F. de Moura, A. V. Malyshev, M. L. Lyra, V. A. Malyshev, and F. Domínguez-Adame, Localization properties of a one-dimensional tight-binding model with nonrandom long-range intersite interactions, Phys. Rev. B 71, 174203 (2005).

[38] M. Gärttner, S. V. Syzranov, A. M. Rey, V. Gurarie, and L. Radzihovsky, Disorder-driven transition in a chain with powerlaw hopping Phys. Rev. B 92, 041406(R) (2015).

[39] K. S. Tikhonov and A. D. Mirlin, Many-body localization transition with power-law interactions: Statistics of eigenstates, Phys. Rev. B 97, 214205 (2018).

[40] N. Y. Yao, C. R. Laumann, S. Gopalakrishnan, M. Knap, M. Müller, E. A. Demler, and M. D. Lukin, Many-Body Localization in Dipolar Systems, Phys. Rev. Lett. 113, 243002 (2014).

[41] D. M. Basko, I. L. Aleiner, and B. L. Altshuler, Metal insulator transition in a weakly interacting many-electron system with localized single-particle states, Ann. Phys. 321, 1126 (2006).

[42] R. Nandkishore and D. A. Huse, Many-body localization and thermalization in quantum statistical mechanics, Annu. Rev. Condens. Matter Phys. 6, 15 (2015).

[43] D. A. Abanin and Z. Papić, Recent progress in many-body localization, Ann. Phys. 529, 1700169 (2017).

[44] D. A. Abanin, E. Altman, I. Bloch, and M. Serbyn, Colloquium: Many-body localization, thermalization, and entanglement, Rev. Mod. Phys. 91, 021001 (2019).

[45] A. L. Burin and L. A. Maksimov, Localization and delocalization of particles in disordered lattice with tunneling amplitude with $r^{-3}$ decay, JETP Lett. 50, 338 (1989).

[46] A. L. Burin, Energy delocalization in strongly disordered systems induced by the long-range many-body interaction, arXiv:cond-mat/0611387.

[47] S. V. Syzranov, V. Gurarie, and L. Radzihovsky, Multifractality at non-Anderson disorder-driven transitions in Weyl semimetals and other systems, Ann. Phys. 373, 694 (2016).

[48] F. Evers and A. D. Mirlin, Anderson transitions, Rev. Mod. Phys. 80, 1355 (2008).

[49] K. B. Efetov, Supersymetry in Disorder and Chaos (Cambridge University Press, New York, 1999).

[50] D. B. Gutman, I. V. Protopopov, A. L. Burin, I. V. Gornyi, R. A. Santos, and A. D. Mirlin, Energy transport in the Anderson insulator, Phys. Rev. B 93, 245427 (2016).

[51] See Supplemental Material at http://link.aps.org/supplemental/ 10.1103/PhysRevResearch.1.032035 for the discussion of duality in a continuous potential.

[52] L. Fleishman and P. W. Anderson, Interactions and the Anderson transition, Phys. Rev. B 21, 2366 (1980).

[53] K. Kobayashi, T. Ohtsuki, K.-I. Imura, and I. F. Herbut, Density of States Scaling at the Semimetal to Metal Transition in Three Dimensional Topological Insulators, Phys. Rev. Lett. 112, 016402 (2014).

[54] S. V. Syzranov, V. Gurarie, and L. Radzihovsky, Unconventional localization transition in high dimensions, Phys. Rev. B 91, 035133 (2015).

[55] P. Goswami and S. Chakravarty, Quantum Criticality between Topological and Band Insulators in 3+1 Dimensions, Phys. Rev. Lett. 107, 196803 (2011).

[56] J. H. Pixley, D. A. Huse, and S. Das Sarma, Rare-RegionInduced Avoided Quantum Criticality in Disordered ThreeDimensional Dirac and Weyl Semimetals, Phys. Rev. X 6, 021042 (2016).

[57] B. Sbierski, K. S. C. Decker, and P. W. Brouwer, Weyl node with random vector potential, Phys. Rev. B 94, 220202(R) (2016).

[58] S. Liu, T. Ohtsuki, and R. Shindou, Effect of Disorder in Three Dimensional Layered Chern Insulator, Phys. Rev. Lett. 116, 066401 (2016).

[59] T. Louvet, D. Carpentier, and A. A. Fedorenko, New quantum transition in Weyl semimetals with correlated disorder, Phys. Rev. B 95, 014204 (2017).

[60] F. Wegner, Bounds on the density of states in disordered systems, Z. Phys. B 44, 9 (1981).

[61] I. M. Suslov, Density of states near an Anderson transition in four-dimensional space: Lattice model, Sov. Phys. JETP 79, 307 (1994).

[62] R. Nandkishore, D. A. Huse, and S. L. Sondhi, Rare region effects dominate weakly disordered 3D Dirac points, Phys. Rev. B 89, 245110 (2014).

[63] J. H. Pixley, Y.-Z. Chou, P. Goswami, D. A. Huse, R. Nandkishore, L. Radzihovsky, and S. Das Sarma, Singleparticle excitations in disordered Weyl fluids, Phys. Rev. B 95, 235101 (2017).

[64] V. Gurarie, Theory of avoided criticality in quantum motion in a random potential in high dimensions, Phys. Rev. B 96, 014205 (2017).

[65] M. Buchhold, S. Diehl, and A. Altland, Vanishing Density of States in Weakly Disordered Weyl Semimetals, Phys. Rev. Lett. 121, 215301 (2018).

[66] M. Buchhold, S. Diehl, and A. Altland, Nodal points of Weyl semimetals survive the presence of moderate disorder, Phys. Rev. B 98, 205134 (2018).

[67] B. Sbierski, G. Pohl, E. J. Bergholtz, and P. W. Brouwer, Quantum Transport of Disordered Weyl Semimetals at the Nodal Point, Phys. Rev. Lett. 113, 026602 (2014).

[68] J. H. Pixley, P. Goswami, and S. Das Sarma, Anderson Localization and the Quantum Phase Diagram of Three Dimensional Disordered Dirac Semimetals, Phys. Rev. Lett. 115, 076601 (2015).

[69] S. Bera, J. D. Sau, and B. Roy, Dirty Weyl semimetals: Stability, phase transition, and quantum criticality, Phys. Rev. B 93, 201302(R) (2016).

[70] C. Wang, P. Yan, and X. R. Wang, Non-Wigner-Dyson level statistics and fractal wave function of disordered Weyl semimetals, Phys. Rev. B 99, 205140 (2019). 